\title{
Coordinated beamforming design for multicell systems with transceiver impairments: from perspective of spectral efficiency to energy efficiency
}

\author{
Yongming Huang ${ }^{1 *}$, Fan Zhang ${ }^{1}$, Shiwen $\mathrm{He}^{1,2}$, Shi Jin $^{1}$ and Luxi Yang ${ }^{1}$
}

\begin{abstract}
Physical transceiver implementations for wireless communication systems usually suffer from transmit-radio frequency (Tx-RF) and receiver-RF (Rx-RF) impairments. In this paper, we aim to design efficient coordinated beamforming for multicell multiuser multi-antenna systems by fully taking into account the residual transceiver impairments. Our design objectives include both spectral efficiency and energy efficiency. In particular, we first derive the closed-form expression of the mean square error (MSE) which includes the impact of transceiver impairments. Based on that, we propose an alternating optimization algorithm to solve the coordinated multicell beamforming problems with the goal of minimizing the worst user MSE, and the sum MSE. Then, by exploiting the relationship between the minimum mean square error (MMSE) and the achievable rate, we develop a new algorithm to address the sum rate maximization problem. This approach is further generalized to solve the more intractable energy efficiency optimization problem. We prove that all the proposed iterative algorithms guarantee to converge to a stationary point. Numerical results show that our proposed schemes achieve a better performance than conventional coordinated beamforming algorithms that were designed ignoring the transceiver impairments.
\end{abstract}

Keywords: Coordinated beamforming; Transceiver impairment; Spectral efficiency; Energy efficiency

\section{Introduction}

High inter-cell interference (ICI) is a limiting factor of current wireless cellular networks, which would severely degrade the performance of cell-edge users. To address this issue, multicell cooperation has emerged as a powerful tool and recently has drawn a lot of research interest in both the industry [1] and the academia [2].

Coordinated or cooperative beamforming, where multiantenna reprocessing at neighboring base stations (BSs) are designed cooperatively, has been extensively studied in the existing literatures such as [3-9] and thereof. To be more specific, the transmit power minimization problem subject to signal-to-interference-and-noise-ratio (SINR) constraints at the remote users was addressed based on

\footnotetext{
${ }^{*}$ Correspondence: huangym@seu.edu.cn

1 School of Information Science and Engineering, Southeast University, Nanjing 210096, China

Full list of author information is available at the end of the article
}

the uplink-downlink duality theorem for multicell multiuser downlink systems [3]. To maximize the minimum user rate, we proposed an efficient iterative algorithm to optimize the downlink beamforming and power allocation in time-division-duplex systems [4], which only requires limited signaling exchange between BSs. Unlike the aforementioned references where a coordinated beamforming algorithm was designed assuming a fixed coordinated BS cluster, in [5], the authors investigated a novel multicell coordinated beamforming framework with dynamic cooperation clusters where each user is jointly served by a small set of BSs. In [6], the number of transmitted streams and the user scheduling in all cells were jointly optimized in order to maximize a network utility function accounting for fairness among users. Based on a novel virtual SINR framework, a distributed precoding design method which needs only local channel state information (CSI) was proposed for a coordinated joint transmission system [7]. Recently, taking the user fairness in account,

\section{Springer}

(c) 2015 Huang et al. Open Access This article is distributed under the terms of the Creative Commons Attribution 4.0 International License (http://creativecommons.org/licenses/by/4.0), which permits unrestricted use, distribution, and reproduction in any medium, provided you give appropriate credit to the original author(s) and the source, provide a link to the Creative Commons license, and indicate if changes were made. The Creative Commons Public Domain Dedication waiver (http:// creativecommons.org/publicdomain/zero/1.0/) applies to the data made available in this article, unless otherwise stated. 
a decentralized coordinated beamforming algorithm was proposed to achieve the Pareto boundary of user rate tuples [8]. Further considering a massive MIMO case, an efficient coordinated multicell beamforming scheme was developed by exploiting the asymptotic behavior of massive MIMO channels, which could asymptotically achieve the optimal performance with limited intercell coordination [9].

More recently, improving energy efficiency has become increasingly important for cellular networks because the growing demand of ubiquitous multimedia communications will accompany with a rapid increase of energy consumption. Besides designing effective resource allocation approaches to improve the energy efficiency, it has been shown that coordinated MIMO transmission is also able to greatly improve the energy efficiency while retaining the performance of spectral efficiency [10-16], especially when the number of transmit antennas is large. In particular, in [10], the energy-efficient multiuser beamforming optimization problem was solved by approximating the objective with a convex and tight upper bound function. In [11], the solution to the energy efficiency optimization problem was achieved by solving three slightly tractable subproblems and choosing the best one. In [12], a zero-gradient-based energy-efficient iterative approach was developed which was guaranteed to converge to a local maximum for MIMO interference channel. In [13], an uplink-downlink duality-based beamforming approach was proposed to achieve a reasonable SE and EE tradeoff in a multiuser downlink system. Focusing on the downlink of multicell multiuser systems, we addressed in [14, 15] the energy-efficient beamforming problem with per-BS power constraints by using jointly the fractional programming and the relationship between the user rate and the minimum mean square error (MMSE). Furthermore, we developed a distributed solution to energy-efficient multicell beamforming in [16].

Though the above developed schemes promise to achieve huge gain in theory, the performance obtained in practical cellular systems is limited by various nonidealities, such as CSI uncertainty and transceiver impairments. For the CSI uncertainty, some robust algorithms were developed recently [17-21] by taking into account the nonideal CSI in the beamforming design. In particular, in [17], a robust coordinated beamforming algorithm was developed with the goal of minimizing transmit power subject to probability constraint of quality of service (QoS). The max-min worst-case SINR optimization problem was investigated in $[19,20]$ and solved by the wellknown bisection method. The robust weighted sum MSE minimization problem with per-BS power constraints was addressed in [21] for multicell downlink joint transmission systems. As for the transceiver, impairments suffered in practical systems, its effect on the performance of MIMO orthogonal frequency division multiplexing (MIMO-OFDM) communication systems was analyzed in $[22,23]$. Later, the effect of residual Tx-RF impairments that defy proper compensation on channel capacity and error-rate performance was disclosed in [24]. To combat transceiver impairments, a common way is to assume that the impairment is signal-independent and thus its addressing methods can be devised separately from the beamforming design in multi-antenna systems. Unfortunately, physical hardware implementations of radio frequency transceivers usually suffer from signal-dependent impairments, including the impairments from nonlinear power amplifier, phase noise and IQ-imbalance [25]. These impairments have a minor effect on point-to-point systems with low-order modulation and operating at low SNRs [26, 27]. For these reasons, transceiver impairments have preciously attracted much less attention in the beamforming design [28, 29]. However, in contrast to point-topoint systems, its caused peformance degrade would be particularly severe in a multicell multiuser system [30-33].

In this paper, we focus on a coordinated beamforming design for the multicell multiuser multi-antenna downlink system with residual transceiver impairments. In order to fully take into account the transceiver impairments, we first derive the closed-form expression of its mean square error (MSE) which includes the impact of residual transceiver impairments. Then, we propose an iterative beamforming scheme with proved convergence to solve the problems including the worst user MSE minimization problem and the sum MSE minimization problem. Furthermore, we exploit the relationship between the MMSE and the achievable rate to find an efficient solution to the system sumrate maximization problem. This approach is also generalized to tackle the more intractable energyefficient coordinated beamforming problem. Numerical results show that our proposed schemes achieve better performance than conventional schemes which do not consider transceiver impairments in the beamforming design.

\section{System model}

Consider $M$ cells allowing to perform coordinated beamforming for the downlink system, where each cell consists of an $N_{t}$-antenna base station (BS), and $I$ single-antenna users. For convenience, let User- $(i, j)$ denote the $j$ th user in the $i$ th cell, BS- $i$ denote the BS in the $i$ th cell. The received signal of User- $(i, j)$ is given by

$$
y_{i, j}=\sum_{m=1}^{M} \mathbf{h}_{m, i, j}^{H}\left(\sum_{k=1}^{I} \mathbf{w}_{m, k} x_{m, k}+\mathbf{z}_{m}^{(t)}\right)+z_{i, j}^{(r)}
$$

where, $\mathbf{h}_{m, i, j} \in \mathbb{C}^{N_{t} \times 1}$ denotes the channel vector from BS$m$ to User- $(i, j), x_{i, j}$ denotes the zero mean unit variance 
circular-symmetric complex Gaussian signal for User$(i, j), \mathbf{w}_{i, j} \in \mathbb{C}^{N_{t} \times 1}$ denotes the beamforming vector for User- $(i, j), \mathbf{z}_{m}^{(t)}$ denotes the transmitter distortion at BS$m, z_{i, j}^{(r)}$ denotes receiver distortion which absorbs thermal noise at User- $(i, j)$. Without loss of generality, in the rest of this paper, we assume that $x_{i, j}, \mathbf{z}_{m}^{(t)}$ and $z_{i, j}^{(r)}$ are mutually independent, and $\mathbf{W}_{m}=\left[\mathbf{w}_{m, 1}, \cdots, \mathbf{w}_{m, I}\right]$ denotes the cascade beamforming matrix at BS- $m$. Previous study has shown that residual transceiver impairments can be well modeled as Gaussian noise with a variance which increases with the power of transmit signal [30], as these impairments are usually followed by calibration and compensation, behaving as the aggregate residual of many distortions. In particular, the transmitter distortion is modeled as $\mathbf{z}_{m}^{(t)} \sim \mathcal{C N}\left(0, \mathbf{C}_{m}\right)$ with $^{1}$

$$
\mathbf{C}_{m}=\left[\begin{array}{ccc}
c_{m, 1}^{2} & & \\
& \ddots & \\
& & c_{m, M}^{2}
\end{array}\right], c_{m, n}=\eta\left(\left\|\mathbf{T}_{n} \mathbf{W}_{m}\right\|_{F}\right)
$$

where the square matrix $\mathbf{T}_{n}$ picks out the transmit magnitude at the $n$th antenna, i.e., the $n$th diagonal-element of $\mathbf{T}_{n}$ is one, while all other elements are zero. The monotonically increasing continuous function $\eta(\cdot)$ of the transmit magnitude (in $\sqrt{\mathbf{m W}}$ ) models the characteristics of the impairments and is given as

$$
\eta(x)=\frac{k_{1}}{100} x\left(1+\left(\frac{x}{k_{2}}\right)^{4}\right)[\sqrt{\mathbf{m W}}]
$$

where $x=\left\|\mathbf{T}_{n} \mathbf{W}_{m}\right\|_{F}$ denotes the transmit magnitude of the $n$th transmit antenna of BS- $m$, and $k_{1}, k_{2}$ are model parameters which are closely related to the error vector magnitude (EVM) of corresponding hardware implementation. Usually, EVM is defined as [34]

$$
\mathrm{EVM}_{m, n}=\frac{E\left\{\left|\left[\mathbf{z}_{m}^{(t)}\right]_{n}\right|^{2}\right\}}{E\left\{\left|\left[\sum_{k} \mathbf{w}_{m, k} x_{m, k}\right]_{n}\right|^{2}\right\}}=\left(\frac{\eta\left(\left\|\mathbf{T}_{n} \mathbf{W}_{m}\right\|_{F}\right)}{\left\|\mathbf{T}_{n} \mathbf{W}_{m}\right\|_{F}}\right)^{2}
$$

The impairment of LTE transceiver can be accurately modeled by (3), as shown in Fig. 1 , where $k_{1}=0.43$, $k_{2}=4.6$ [35]. Similarly, the receiver distortion of User$(i, j)$, which mainly suffers from the phase noise and I-Q imbalance, can be modeled as $z_{i, j}^{(r)} \sim \mathcal{C N}\left(0, \sigma_{i, j}^{2}\right)$, which includes thermal noise and transceiver impairments [34], and is given by

$$
\sigma_{i, j}^{2}=\sigma^{2}+v^{2}\left(\sqrt{\sum_{m=1}^{K}\left\|\mathbf{h}_{m, i, j}^{H} \mathbf{W}_{m}\right\|_{F}^{2}}\right)[\sqrt{\mathbf{m V}}]
$$

where $\sigma^{2}$ denotes thermal noise variance, the second term models the receiver impairment characteristics and is a monotonically increasing function of received signal, defined as

$$
v(x)=\frac{k_{3}}{100} x \quad[\sqrt{\mathbf{m W}}]
$$

in which $k_{3}$ is a model parameter and equals EVM \%.

\section{Beamforming design for minmizing MSE}

Conventional coordinated multicell beamforming approaches usually ignore the impact of transceiver impairment to simplify the design. However, in practical systems, the degrade from transceiver impairments can only be partially compensated at the transmitter through calibration and pre-distortion, or at the receiver using sophisticated compensation algorithms. The residual distortion is severe in the multicell multiuser beamforming systems and is signal dependent, especially at high-order modulations and high SNR regime. Motivated by this, here we design coordinated beamforming schemes based on the above system model which properly takes the transceiver impairment into account. Here our design objective is first to minimize the worst user MSE or the sum MSE of all users.

\subsection{Problem formulation}

Consider a linear receiver and the estimated signal of User- $(i, j)$ is denoted as $\widehat{x}_{i, j}=u_{i, j}^{*} y_{i, j}$ with the equalizer $u_{i, j}$ where " $*$ " denotes the conjugate operation. Thus, the MSE of User- $(i, j)$ can be calculated as follows

$$
\begin{aligned}
\operatorname{MSE}_{i, j}= & \mathbb{E}\left\{\left|\widehat{x}_{i, j}-x_{i, j}\right|^{2}\right\}=\mathbb{E}\left\{\hat{x}_{i, j} \hat{x}_{i, j}^{*}\right\}-\mathbb{E}\left\{\hat{x}_{i, j} x_{i, j}^{*}\right\} \\
& -\mathbb{E}\left\{x_{i, j} \hat{x}_{i, j}^{*}\right\}+\mathbb{E}\left\{x_{i, j} x_{i, j}^{*}\right\} \\
= & \left|u_{i, j}\right|^{2} \sum_{m=1}^{K} \sum_{k=1}^{I} \mathbf{h}_{m, i, j}^{H} \mathbf{w}_{m, k} \mathbf{w}_{m, k}^{H} \mathbf{h}_{m, i, j} \\
& +\left|u_{i, j}\right|^{2} \sum_{m=1}^{K} \mathbf{h}_{m, i, j}^{H} \mathbf{C}_{m} \mathbf{h}_{m, i, j}+\left|u_{i, j}\right|^{2} \sigma_{i, j}^{2} \\
& -u_{i, j}^{*} \mathbf{h}_{i, i, j}^{H} \mathbf{w}_{i, j}-\mathbf{w}_{i, j}^{H} \mathbf{h}_{i, i, j} u_{i, j}+1
\end{aligned}
$$

We are now ready to present two optimization objectives to investigate the effect of transceiver impairments on the coordinated beamforming design. The first one is the minimizing maximum MSE optimization problem which is given as

$$
\begin{gathered}
\min _{\mathbf{W}_{i}, u_{i, j}, \forall i, j} \max _{\forall i, j} \operatorname{MSE}_{i, j} \\
\text { s.t. } \operatorname{tr}\left(\mathbf{W}_{i} \mathbf{Q}_{i, k} \mathbf{W}_{i}\right)+\operatorname{tr}\left(\mathbf{Q}_{i, k} \mathbf{C}_{i}\right) \leq q_{i, k}, \forall i, k
\end{gathered}
$$

and the other is the sum MSE minimization problem which is formulated as

$$
\min _{\mathbf{W}_{i}, u_{i, j}, \forall i, j} \sum_{i, j} \operatorname{MSE}_{i, j}
$$

$$
\text { s.t. } \operatorname{tr}\left(\mathbf{W}_{i} \mathbf{Q}_{i, k} \mathbf{W}_{i}\right)+\operatorname{tr}\left(\mathbf{Q}_{i, k} \mathbf{C}_{i}\right) \leq q_{i, k}, \forall i, k
$$




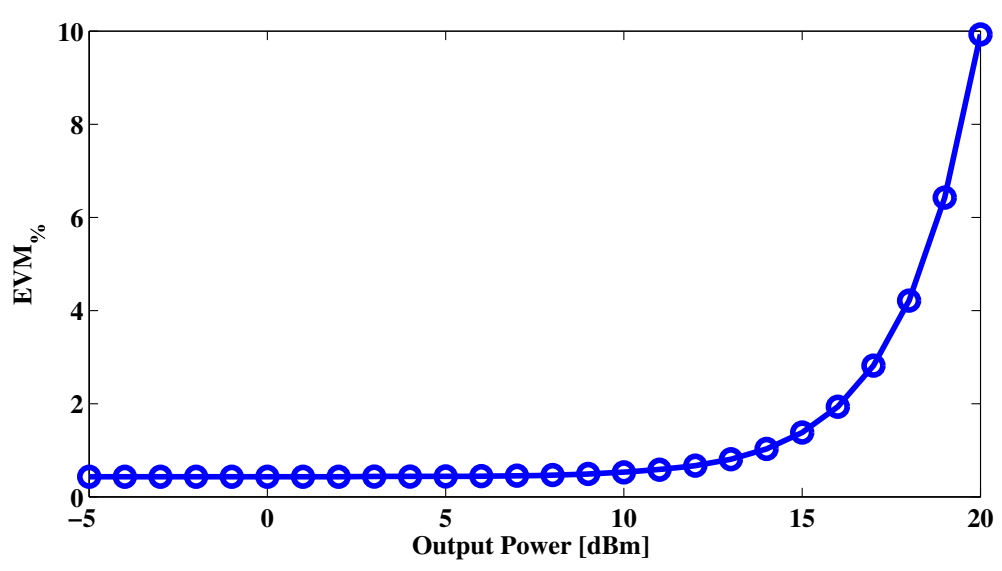

Fig. 1 EVM versus output power for the LTE power amplifier HXG-122+ in [34] using 64-QAM waveforms and a state-of-the-art signal generator

where all $\mathbf{Q}_{i, k} \in \mathbb{C}^{N_{t} \times N_{t}}$ are Hermitian positive semidefinite matrices satisfying $\sum_{k=1}^{L_{i}} \mathbf{Q}_{i, k} \succ \mathbf{0}_{N_{t}}$. Let $L_{i}$ denote the number of the transmit power constraints at BS- $i$. That is, if a total power constraint is adopted, we have $L_{i}=1$ and $\mathbf{Q}_{i, k}=\mathbf{I}_{N_{t}}$, while if per-antenna constraints are used, we have $L_{i}=N_{t}$ and $\mathbf{Q}_{i, k}=\mathbf{T}_{k}, \forall k$.

\subsection{Algorithm design}

Note that although functions $\eta(x)$ defined in (3) and $v(x)$ in (6) are both convex, the expression of the MSE becomes extremely complex if we substitute directly (3) and (6) into (7). To obtain a tractable form of problem (8), we reformulate it as the following form by introducing auxiliary variables $t_{m, n}, r_{i, j}$ and $\tau$ [29]:

$$
\begin{array}{ll} 
& \min _{\text {s.t. }} \operatorname{MSE}_{i, j} \leq \tau, \forall i, j, \\
& \operatorname{tr}\left(\mathbf{W}_{i} \mathbf{Q}_{i, k} \mathbf{W}_{i}\right)+\sum_{n=1}^{N_{t}} \operatorname{tr}\left(\mathbf{Q}_{i, k} T_{n}\right) t_{i, n}^{2} \leq q_{i, k}, \forall i, k, \\
& \eta\left(\left\|\mathbf{T}_{n} \mathbf{W}_{m}\right\|_{F}\right) \leq t_{m, n}, \forall m, n, \\
& v\left(\sqrt{\sum_{m=1}^{M}\left\|\mathbf{h}_{m, i, j}^{H} \mathbf{W}_{m}\right\|_{F}^{2}}\right) \leq r_{i, j}, \forall i, j \\
& t_{i, n} \geq 0, r_{i, j} \geq 0, \forall i, j, n,
\end{array}
$$

where the 3rd and 4th constraint sets are some auxiliary constraints, $t_{m, n}=c_{m, n}=\eta\left(\left\|\mathbf{T}_{n} \mathbf{W}_{m}\right\|_{F}\right), r_{i, j}=$ $v\left(\sqrt{\sum_{m=1}^{M}\left\|\mathbf{h}_{m, i, j}^{H} \mathbf{W}_{m}\right\|_{F}^{2}}\right)$ and $\operatorname{MSE}_{i, j}$ is rewritten as

$$
\begin{aligned}
\operatorname{MSE}_{i, j}= & \sum_{(m, k) \neq(i, j)}\left|u_{i, j}^{*} \mathbf{h}_{m, i, j}^{H} \mathbf{w}_{m, k}\right|^{2}+\sum_{m=1}^{M} \sum_{n=1}^{N_{t}}\left|u_{i, j}^{*} \mathbf{h}_{m, i, j, n}^{*} t_{m, n}\right|^{2} \\
& +\left|u_{i, j}^{*} r_{i, j}\right|^{2}+\left|u_{i, j}^{*} \sigma\right|^{2}+\left|u_{i, j}^{*} \mathbf{h}_{i, i, j}^{H} w_{i, j}-1\right|^{2} .
\end{aligned}
$$

Note that functions $\eta(\cdot)$ and $v(\cdot)$ defined in (3) and (6) all are convex functions, the transmitter impairment constraints $\eta\left(\left\|\mathbf{T}_{n} \mathbf{W}_{m}\right\|_{F}\right) \leq t_{m, n}$ and the receiver impairment constraints $v\left(\sqrt{\sum_{m=1}^{M}\left\|\mathbf{h}_{m, i, j}^{H} \mathbf{W}_{m}\right\|_{F}^{2}}\right) \leq r_{i, j}$ both are convex constraints too. Herein, without loss of generality, we assume that the mentioned functions are convex functions. However, the optimization problem (10) is still non-convex due to the coupling between the optimization variables $W_{i}$ and $u_{i, j}, \forall i, j$. In what follows, we adopt the alternative optimization methods to design an effective optimization algorithm to address this problem. To proceed, we first provide the following lemma.

Lemma 1. For a given beamforming vector $\left\{w_{i, k}\right\}$, the optimal equalizer $u_{i, j}^{o p t}$ of User- $(i, j)$ that minimizing the $M_{S} E_{i, j}$ is given by

$$
\begin{gathered}
u_{i, j}^{o p t}=\frac{\mathbf{h}_{i, i, j}^{H} \mathbf{w}_{i, j}}{\mathbf{B}_{i, j}} \\
\text { where } \mathbf{B}_{i, j}=\sum_{m=1}^{M} \sum_{k=1}^{I} \mathbf{h}_{m, i, j}^{H} \mathbf{w}_{i, j} \mathbf{w}_{i, j}^{H} \mathbf{h}_{m, i, j}+\sum_{m=1}^{M} \mathbf{h}_{m, i, j}^{H} \mathbf{C}_{m} \mathbf{h}_{m, i, j}+\sigma_{i, j}^{2}
\end{gathered}
$$

Proof. From Lagrange KKT conditions, we obtain the derivation of (7) with respect to $u_{i, j}^{*}$ as follows

$$
\frac{\partial \mathrm{MSE}_{\mathrm{i}, \mathrm{j}}}{\partial u_{i, j}^{*}}=u_{i, j} \mathbf{B}_{i, j}-\mathbf{h}_{i, i, j}^{H} \mathbf{w}_{i, j}=0
$$


where $\mathbf{B}_{i, j}=\sum_{m=1}^{M} \sum_{k=1}^{I} \mathbf{h}_{m, i, j}^{H} \mathbf{w}_{i, j} \mathbf{w}_{i, j}^{H} \mathbf{h}_{m, i, j}+\sum_{m=1}^{M} \mathbf{h}_{m, i, j}^{H} \mathbf{C}_{m} \mathbf{h}_{m, i, j}+\sigma_{i, j}^{2}$. Based on that, the optimal equalizer for a fixed beamforming vector, written as (12), can be readily achieved.

On the other hand, for fixed equalizer $u_{i, j}, \forall i, j$, problem (10) can be easily solved by second order conic programming (SOCP) method. Similarly, problem (9) also can be solved using SOCP for a given $u_{i, j}$. Based on above results, an alternating optimization algorithm is proposed to solve problem (8) and problem (9), which is summarized as Algorithm 1 in the following. For convenience, let $\tau$ to be the target value of problem (8) and problem (9).

$\overline{\text { Algorithm } 1 \text { Alternating optimization algorithm for }}$ minimizing MSE

1: Let $n=0$, initialize the equalizer $u_{i, j}^{(n)}$, beamforming $\operatorname{matrix} \mathbf{W}_{i}^{(n)}=0$ and $\tau^{(n)}=0$;

2: Given $u_{i, j}^{(n)}$, solve problem (8) or problem (9), then obtain $\mathbf{W}_{i}^{(n+1)}$ and $\tau^{(n+1)}$;

3: If $\left|\tau^{(n+1)}-\tau^{(n)}\right|<\delta$, where $\delta$ is a stop threshold, then stop, otherwise, let $n=n+1$, update the equalizer $u_{i, j}$ with (12) and $\mathbf{W}_{i}^{(n)}$, then obtain $u_{i, j}^{(n)}$ and go to step 2.

Theorem 1. Algorithm 1 guarantees to converge.

Proof. Algorithm 1 generates a monotonic decreasing sequence while the iteration is running, due to the fact that the updates of the beamforming vectors and the equalizer at steps 2 and 3 all aim to minimize the object value. Moreover, the value of MSE is bounded in practical wireless communication systems. Therefore, the convergence of Algorithm 1 is guaranteed by the monotonic boundary theorem [36].

\section{Beamforming design for maximizing the system sumrate}

System sumrate is an important metric of wireless communication systems. In this section, we design the coordinated beamforming scheme to maximize the system sumrate achieved with transceiver impairments.

\subsection{Problem formulation}

Consider a multicell multiuser downlink system as stated in Section 2. The SINR for User- $(i, j)$ is expressed as

$$
\operatorname{SINR}_{i, j}=\frac{\left|\mathbf{h}_{i, i, j}^{H} \mathbf{w}_{i, j}\right|^{2}}{\sum_{(m, k)}\left|\mathbf{h}_{m, i, j}^{H} \mathbf{w}_{m, k}\right|^{2}-\left|\mathbf{h}_{i, i, j}^{H} \mathbf{w}_{i, j}\right|^{2}+\sum_{m=1}^{K} \sum_{n=1}^{M} \mathbf{h}_{m, i, j}^{H} \mathbf{T}_{n} \mathbf{h}_{m, i, j} t_{m, n}^{2}+\sigma_{i, j}^{2}}
$$

Further, the data rate for User- $(i, j)$ is defined as:

$$
R_{i, j}=\log _{2}\left(1+\operatorname{SINR}_{i, j}\right)
$$

As stated above, our design objective in this section is to maximize the system sumrate. Then, the optimization problem is formulated as

$$
\begin{aligned}
& \max _{\mathbf{W}_{i}, u_{i, j}, \forall i, j} \sum_{i, j} R_{i, j} \\
& \text { s.t. } \operatorname{tr}\left(\mathbf{W}_{i}^{H} \mathbf{Q}_{i, k} \mathbf{W}_{\mathbf{i}}\right)+\operatorname{tr}\left(\mathbf{Q}_{i, k} \mathbf{C}_{i}\right) \leq q_{i, k}, \forall i, k .
\end{aligned}
$$

\subsection{Algorithm design}

Compared with the MSE minimization problem, it is more difficult to directly solve the above sumrate maximization problem (16), due to the non-convex nature of the sumrate objective with respect to the beamforming vectors. To address it, here, we resort to transforming the original problem into a tractable equivalent form using the following lemma [37].

Lemma 2. Assuming that $d$ is an arbitrary integer, $\mathbf{E} \in$ $\mathbb{C}^{d \times d}$ is an arbitrary matrix which satisfies the condition of $\mathbf{E} \succ 0$ and $\operatorname{det}(\mathbf{E}) \leq 1$. If we define a function given by

$$
\varphi(\mathbf{S})=-\operatorname{Tr}(\mathbf{S E})+\log _{2} \operatorname{det}(\mathbf{S})+d
$$

Then, we have

$$
\max _{\mathbf{S} \in \mathbb{C}^{d \times d}, \mathbf{S} \succ 0} \varphi\left(\mathbf{S}^{\text {opt }}\right)=\log _{2} \operatorname{det}\left(\mathbf{E}^{-1}\right)
$$

where $\mathbf{S}^{\text {opt }}=\mathbf{E}^{-1}$.

In order to make use of Lemma 2 to solve the sumrate maximization optimization problem, we introduce a matrix variable $\mathbf{E} \in C^{K I \times K I}$ given as

$$
\mathbf{E}=\left[\begin{array}{cccc}
\operatorname{MSE}_{1,1} & & & \mathbf{0} \\
& \operatorname{MSE}_{1,2} & & \\
& & \ddots & \\
& & & \operatorname{MSE}_{K, I}
\end{array}\right]_{K I \times K I}
$$

where $d=K I$ donotes the total number of data streams in the system. Furthermore, in the considered optimizaiton problem that has taken the transceiver impairment into account, it is easy to understand that the mean square error of User- $(i, j)$ has $0<\operatorname{MSE}_{i, j} \leq \mathbf{E}\left\{\left|x_{i, j}\right|^{2}\right\}=1$ which satisfies the restriction $\operatorname{det}|\mathbf{E}|<1$ put forward by Lemma 2.

Lemma 3. When using the MMSE receiver, the maximum value of the function $\varphi(\mathbf{S})=-\operatorname{Tr}(\mathbf{S E})+\log _{2}|\mathbf{S}|+K I$ defined in (17) is equal to the sumrate calculated as

$$
\sum_{i, j} R_{i, j}=\max _{\mathbf{S} \in \mathbb{C}^{d \times d}, \mathbf{S} \succ 0} \varphi(\mathbf{S})
$$

where $\mathbf{S}$ is auxiliary matrix variable, given as

$$
\mathbf{S}=\left[\begin{array}{cccc}
S_{1,1} & & & \mathbf{0} \\
& S_{1,2} & & \\
& & \ddots & \\
& & & S_{K, I}
\end{array}\right]
$$


Proof: By substituting (12) into (7), the MSE of User$(i, j)$ is rewritten as

$$
\operatorname{MSE}_{i, j}=\frac{\sum_{(m, k) \neq(i, j)}\left|\mathbf{h}_{m, i, j}^{H} \mathbf{w}_{m, k}\right|^{2}+\sum_{m=1}^{K} \sum_{n=1}^{M} \mathbf{h}_{m, i, j}^{H} \mathbf{T}_{n} \mathbf{h}_{m, i, j} t_{m, n}^{2}+\sigma_{i, j}^{2}}{\sum_{(m, k)}\left|\mathbf{h}_{m, i, j}^{H} \mathbf{w}_{m, k}\right|^{2}+\sum_{m=1}^{K} \sum_{n=1}^{M} \mathbf{h}_{m, i, j}^{H} \mathbf{T}_{n} \mathbf{h}_{m, i, j} t_{m, n}^{2}+\sigma_{i, j}^{2}}
$$

From equation (7), (14), and (22), we notice that

$$
R_{i, j}=\log _{2}\left(1+\operatorname{SINR}_{i, j}\right)=\log _{2}\left(\operatorname{MSE}_{i, j}^{-1}\right)
$$

According to Lemma 2, the max value of function $\varphi(\mathbf{S})=$ $-\operatorname{Tr}(\mathbf{S E})+\log _{2} \operatorname{det}(\mathbf{S})+d$ is obtained by $\mathbf{S}^{\text {opt }}=\mathbf{E}^{-1}$. Then, we have

$$
\varphi_{\max }(\mathbf{S})=\log _{2}\left|\mathbf{S}^{\text {opt }}\right|=\log _{2}\left|\mathbf{E}^{-1}\right|=\sum_{i, j} \log _{2} \mathrm{MSE}_{i, j}^{-1}=\sum_{i, j} R_{i, j} .
$$

Based on the results above, the sumrate maximization optimization problem can be transformed into the following form:

$$
\begin{aligned}
& \max _{\mathbf{W}_{i}, S_{i, j}, u_{i, j,}, \forall i, j}-\operatorname{Tr}(\mathbf{S E})+\log |\mathbf{S}|+K I \\
& \text { s.t. } \operatorname{tr}\left(\mathbf{W}_{i}^{H} \mathbf{Q}_{i, k} \mathbf{W}_{\mathbf{i}}\right)+\operatorname{tr}\left(\mathbf{Q}_{i, k} \mathbf{C}_{i}\right) \leq q_{i, k}, \forall i, k
\end{aligned}
$$

This equivalent optimization problem is still hard to solve due to the coupling between optimization variables $\mathbf{W}_{i}$ and $u_{i, j}$. To find a tractable form, we further introduce auxiliary variables $t_{m, n}$ and $r_{i, j}$ to reconstruct its form, given by

$$
\begin{gathered}
\max _{\mathbf{W}_{i}, S_{i, j}, u_{i, j}, t_{i, n}, r_{i, j} \forall i, j} \quad-\operatorname{Tr}(\mathbf{S E})+\log |\mathbf{S}|+K I \\
\text { s.t. } t_{i, n} \geq 0, \quad r_{i, j} \geq 0 \quad \forall i, j, n \\
\operatorname{tr}\left(\mathbf{W}_{i}^{H} \mathbf{Q}_{i, k} \mathbf{W}_{\mathbf{i}}\right)+\sum_{n} \operatorname{tr}\left(\mathbf{Q}_{i, k} \mathbf{T}_{n}\right) t_{i, n}^{2} \leq q_{i, k} \quad \forall i, k \\
\eta\left(\left\|\mathbf{T}_{n} \mathbf{W}_{m}\right\|_{F}\right) \leq t_{m, n} \quad \forall m, n \\
v\left(\sqrt{\sum_{m=1}^{M}\left\|\mathbf{h}_{m, i, j}^{H} \mathbf{W}_{m}\right\|_{F}^{2}}\right) \leq r_{i, j} \quad \forall i, j
\end{gathered}
$$

where $t_{m, n}=c_{m, n}=\eta\left(\left\|\mathbf{T}_{n} \mathbf{W}_{m}\right\|_{F}\right), r_{i, j}^{2}=$ $v^{2}\left(\sqrt{\sum_{m}\left\|\mathbf{h}_{m, i, j}^{H} \mathbf{W}_{m}\right\|_{F}^{2}}\right)$. It is easy to verify that, when $\eta(\cdot)$ and $v(\cdot)$ are convex functions, the transmitter impairment constraints $\eta\left(\left\|\mathbf{T}_{n} \mathbf{W}_{m}\right\|_{F}\right) \leq t_{m, n}$ and the receiver impairment constraints $v\left(\sqrt{\sum_{m}\left\|\mathbf{h}_{m, i, j}^{H} \mathbf{W}_{m}\right\|_{F}^{2}}\right) \leq r_{i, j}$ are both convex constraints.

In what follows, we leverage the block coordinate descent method to solve problem (26). In particular, the update of $\mathbf{S}$ is calculated as $\mathbf{S}^{o p t}=\mathbf{E}^{-1}$ by fixing other variables, and the update of the equalizer $u_{i, j}$ is given by (12). Given $\mathbf{S}, u_{i, j}$, then we solve optimization problem (26) using SOCP method to update $\mathbf{W}_{i}$. The whole iterative optimization process of the proposed sumrate maximization method is summarized in Algorithm 2, as shown in the following table, where $\varphi=-\operatorname{Tr}(\mathbf{S E})+\log _{2}|\mathbf{S}|+$ $K I$ denotes the target value of the convex optimization problem. Threshold $\delta$ suggests the updating convergence degree of the optimization target $\varphi$. The convergence of Algorithm 2 can be guaranteed by the monotonic boundary theorem similar to Algorithm 1 explained before.

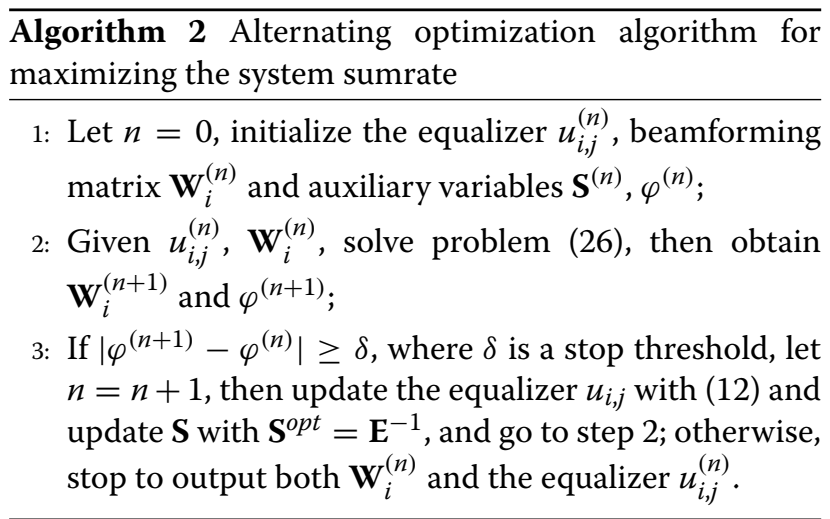

\section{Energy-efficient beamforming design}

Energy efficiency has become increasingly important for cellular networks because the growing demand of ubiquitous multimedia communications will accompany with a rapid increase of energy consumption. In this section, we investigate the multicell cordinated beamforming design aiming at maximizing the system energy efficiency with hardware-impaired transceivers.

\subsection{Problem formulation}

In order to obtain a balance between the sum rate and the total power consumption, we adopt the system energy efficiency optimization criterion defined as

$$
\begin{gathered}
\max _{\mathbf{W}_{i}, \forall i} \sum_{i=1}^{M} \sum_{j=1}^{I} R_{i, j} / \sum_{i=1}^{M} P_{i}^{c}, \\
\text { s.t. } \sum_{j=1}^{I}\left\|\mathbf{w}_{i, j}\right\|^{2}+\operatorname{Tr}\left(\mathbf{C}_{i}\right) \leq P_{i}, \forall i
\end{gathered}
$$

where $P_{i}^{c}$ denotes the total power consumption at BS- $i$ and is calculated as

$$
P_{i^{c}}=\vartheta_{i} \sum_{j=1}^{I}\left\|\mathbf{w}_{i, j}\right\|^{2}+\operatorname{Tr}\left(\mathbf{C}_{i}\right)+M P_{c}+P_{0},
$$


$P_{i}$ is power constraint of BS- $i, P_{c}$ is the constant circuit power consumption per antenna, $P_{0}$ is the basic power consumed at the BS and is independent of the number of transmit antennas, and $\vartheta_{i} \geq 1$ is a constant which accounts for the inefficiency of power amplifier [38].

Note that the coupling of optimization variables leads to that problem (27), becomes non-convex, and therefore is difficult to solve directly. Moreover, the fractional form in the objective function (27) makes the problem more intractable.

\subsection{Algorithm design}

According to Lemma 3, by substituting (20) into the objective function of problem (27), we can transform the optimization problem into the following form:

$$
\begin{aligned}
& \max _{\mathbf{w}_{i}, S_{i, j}, u_{i, j}, \forall i, j}(-\operatorname{Tr}(\mathbf{S E})+\log |\mathbf{S}|+K I) / \sum_{i=1}^{M} P_{i}^{c} \\
& \text { s.t. } \sum_{j=1}^{I}\left\|\mathbf{w}_{i, j}\right\|^{2}+\operatorname{Tr}\left(\mathbf{C}_{i}\right) \leq P_{i}, \forall i
\end{aligned}
$$

Next we focus on finding the solution to problem (28), which equivalently solves the original problem (27). It is easy to see that problem (28) belongs to a classical fractional programming problem. In addition, the works in $[15,39,40]$ have shown that it is equivalent to looking up a value of $\alpha$ such that the optimal objective value of the following optimization problem (29) equals to zero.

$$
\begin{aligned}
& \max _{\mathbf{w}_{i}, S_{i, j}, u_{i, j}, \forall i, j}(-\operatorname{Tr}(\mathbf{S E})+\log |\mathbf{S}|+M I)-\alpha \sum_{i=1}^{M} P_{i}^{c} \\
& \text { s.t. } \sum_{j=1}^{I}\left\|\mathbf{w}_{i, j}\right\|^{2}+\operatorname{Tr}\left(\mathbf{C}_{i}\right) \leq P_{i}, \forall i
\end{aligned}
$$

The optimal value of $\alpha$ can be obtained via iteratively solving problem (29) with fixed $\alpha$ and updating the value of $\alpha$ with gradient method $[15,39,40]$.

In what follows, we attempt to develop an efficient algorithm to solve the above problem with fixed $\alpha$. The cost function of problem (29) is concave in each of the optimization variables $\mathbf{W}_{i}, u_{i, j}, \mathbf{S}$. To address this issue, we propose to use the block coordinate descent method to solve (29). To be more specific, we solve the problem by sequentially fixing two of the three variables $\mathbf{W}_{i}, u_{i, j}, \mathbf{S}$ and updating the third. For given $\mathbf{W}_{i}$, the optimal solutions of $u_{i, j}$ and $\mathbf{S}$ are given by (12) and $\mathbf{S}^{\text {opt }}=\mathbf{E}^{-1}$, respectively. With fixed $u_{i, j}$ and $\mathbf{S}$, by dropping the constant item and rearranging the remaining terms using the properties of the trace, problem (29) can be reformulated as (30),

$$
\begin{array}{r}
\min _{\mathbf{w}_{i, t}, t_{i, n}, r_{i, j}} \sum_{i, j} s_{i, j}\left\{\sum_{(m, k) \neq(i, j)}\left|u_{i, j}^{*} \mathbf{h}_{m, i, j}^{H} \mathbf{w}_{m, k}\right|^{2}+\sum_{m=1}^{M} \sum_{n=1}^{N_{t}}\left|u_{i, j}^{*} \mathbf{h}_{m, i, j, n}^{H} t_{m, n}\right|^{2}\right. \\
\left.+\left|u_{i, j}^{*} r_{i, j}\right|^{2}+\left|u_{i, j}^{*} \mathbf{h}_{i, i, j}^{H} \mathbf{w}_{i, j}-1\right|^{2}\right\}+\alpha \sum_{i=1}^{M}\left(\vartheta_{i} \sum_{j=1}^{I}\left\|\mathbf{w}_{i, j}\right\|^{2}+\sum_{n=1}^{N_{t}} t_{i, n}^{2}\right) \\
\text { s.t. } \sum_{j=1}^{I}\left\|\mathbf{w}_{i, j}\right\|^{2}+\sum_{n=1}^{N_{t}} t_{i, n}^{2} \leq P_{i} \quad \forall i, \quad \eta\left(\left\|\mathbf{T}_{n} \mathbf{W}_{m}\right\|_{F}\right) \leq t_{m, n} \quad \forall m, n, \\
v\left(\sqrt{\sum_{m=1}^{M}\left\|\mathbf{h}_{m, i, j}^{H} \mathbf{W}_{m}\right\|_{F}^{2}}\right) \leq r_{i, j} \quad \forall i, j
\end{array}
$$

where $t_{m, n}=c_{m, n}=\eta\left(\left\|\mathbf{T}_{n} \mathbf{W}_{m}\right\|_{F}\right), r_{i, j}^{2}=$ $v^{2}\left(\sqrt{\sum_{m}\left\|\mathbf{h}_{m, i, j}^{H} \mathbf{W}_{m}\right\|_{F}^{2}}\right)$. Since $\eta(\cdot)$ and $v(\cdot)$ are convex, problem (30) is convex and can be solved by generalpurpose interior-point methods. Based on the above analysis, the detailed steps that are used to solve problem (28) are summarized as Algorithm 3, where $\rho$ denotes the objective function value of problem (29).

\section{Algorithm 3 Energy-efficient optimization algorithm \\ 1: Choose arbitrarily $\mathbf{W}_{i}^{(n)}$ such that it satisfies the power constraints, and compute $u_{i, j}^{(n)}$ and $\mathbf{S}^{(n)}$ with $\mathbf{W}_{i}^{(n)}$, (12), and $\mathbf{S}^{\text {opt }}=\mathbf{E}^{-1}$, respectively. Let $\rho^{(n)}=0$.}

2: Compute the value of $\alpha$ with $u_{i, j}^{(n)}, \mathbf{S}^{(n)}$, and $\mathbf{W}_{i}^{(n)}$ and the following equation

$$
\alpha=(-\operatorname{Tr}(\mathbf{S E})+\log |\mathbf{S}|+M I) / \sum_{i=1}^{M} P_{i}^{c}
$$

then obtain $\alpha^{(n)}$.

3: Update $\mathbf{W}_{i}$ with $u_{i, j}^{(n)}, \mathbf{S}^{(n)}$ and $\alpha^{(n)}$ via solving problem (30), then obtain $\mathbf{W}_{i}^{(n+1)}$.

4: Update $u_{i, j}$ with (12) and $\mathbf{W}_{i}^{(n+1)}$, then obtain $u_{i, j}^{(n+1)}$.

5: Update $\mathbf{S}$ with $\mathbf{S}^{\text {opt }}=\mathbf{E}^{-1}, \mathbf{W}_{i}^{(n+1)}$ and $u_{i, j}^{(n+1)}$, then obtain $\mathbf{S}^{(n+1)}$.

6: Compute $\rho$ with $u_{i, j}^{(n+1)}, \mathbf{S}^{(n+1)}$, and $\mathbf{W}_{i}^{(n+1)}$, then obtain $\rho^{(n+1)}$. If $\left|\rho^{(n+1)}-\rho^{(n)}\right| \leq \eta$, where $\eta$ is a predefined value, then go to 7 , otherwise, let $u_{i, j}^{(n)}=$ $u_{i, j}^{(n+1)}, \mathbf{S}^{(n)}=\mathbf{S}^{(n+1)}$, and $\mathbf{W}_{i}^{(n)}=\mathbf{W}_{i}^{(n+1)}$, then go to step 3.

7: If $\left|\rho^{(n+1)}\right| \leq \eta$, then output $\mathbf{W}_{i}^{(n+1)}$ and $\alpha^{(n)}$, otherwise update the value of the $\alpha^{(n)}$ with $u_{i, j}^{(n+1)}, \mathbf{S}^{(n+1)}$, and $\mathbf{W}_{i}^{(n+1)}$ and (31), let $u_{i, j}^{(n)}=u_{i, j}^{(n+1)}, \mathbf{S}^{(n)}=\mathbf{S}^{(n+1)}$, $\mathbf{W}_{i}^{(n)}=\mathbf{W}_{i}^{(n+1)}$, and $\rho^{(n)}=\rho^{(n+1)}$, then go to step 3. 
Remark. The objective value of problem (29) with fixed $\alpha$ generated by the alternating maximization of steps 35 in Algorithm 3 are monotonically increasing, and the objective function can be upper-bound. Thus, the convergence of the alternating maximization of steps $3-5$ in Algorithm 3 can be guaranteed with the monotonic convergence theorem [41]. In the results obtained in [15, 39, 40], one can see that the convergence of Algorithm 3 is also guaranteed. In particular, based on the conclusion given by [41, Prop. 5], it is easy to prove that the converged solution is a stationary point of problem (29).

\section{Simulation results}

In this section, the performances of the proposed coordinated beamforming schemes are investigated via numerical simulations for a multicell multiuser scenario as shown in Fig. 2. The considered system consists of 3 cells with 4 antennas at each BS $\left(M=3, N_{t}=4\right)$. The inter-BS distance is $1 \mathrm{~km}$ and each user has at least $400 \mathrm{~m}$ distance from its serving BS. The channel vector $\mathbf{h}_{m, i, j}$ from BS- $m$ to User- $(i, j)$ is generated based on the formulation $\mathbf{h}_{m, i, j} \triangleq \theta_{m, i, j} \mathbf{h}_{m, i, j}^{w}$, where $\mathbf{h}_{m, i, j}^{w}$ denotes the small scale fading coefficient and is assumed to be Gaussian distributed with zero mean and identity covariance matrix, and $\theta_{m, i, j}$ denotes the large scale fading factor which in decibels is given as $10 \log _{10}\left(\theta_{m, i, j}\right)=-38 \log _{10}\left(d_{m, i, j}\right)-$ $34.5+\eta_{i, j}$, where $\eta_{i, j}$ represents the shadow fading in decibels and follows the distribution $\mathcal{N}(0,8)$ and $d_{m, i, j}$ denotes the distance between BS- $m$ and User- $(i, j)$. The

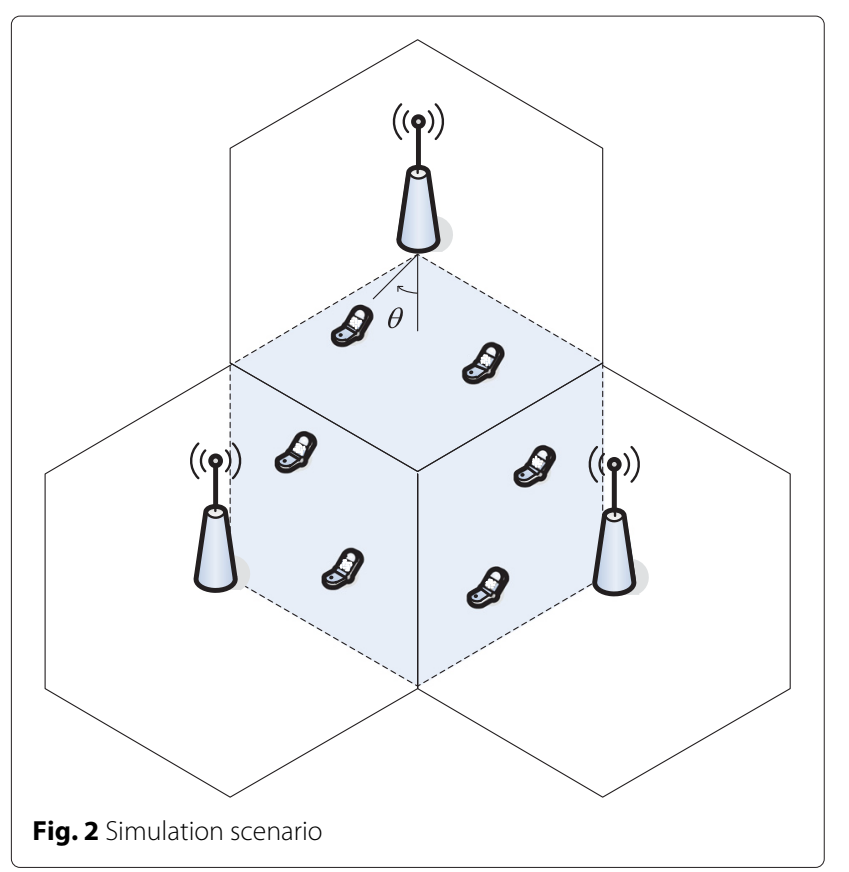

noise figure at each user terminal is $9 \mathrm{~dB}$ [42]. Per-array power constraints of $18.2 \mathrm{dBm}$ per subcarrier (i.e., uniform allocation of $46 \mathrm{dBm}$ ). The system is best described as a simplified version of Case 1 in the 3GPP LTE standard [43].

\subsection{Numerical evaluation for Algorithm 1}

The performances of the proposed multicell multiuser beamforming schemes for minimizing the worst user MSE, minimizing the sum MSE, and maximizing the system sumrate are investigated via numerical simulations. For comparison, we also evaluate the performances of the traditional optimization algorithm without considering the transceiver impairment $\left(k_{1}=0, k_{2}=+\infty, k_{3}=0\right)$.

Figure $3 \mathrm{a}, \mathrm{b}$ show the convergence behavior of Algorithm 1 to solve problem (8) and problem (9) respectively under a few random channel realizations. All simulation results show that Algorithm 1 generates a monotonic decreasing sequence while the iteration is running, by which the convergence of Algorithm 1 can be guaranteed by the monotonic boundary theorem. It is also shown that Algorithm 1 can always converge to a stable point within a couple of iterations.

Figure $4 \mathrm{a}, \mathrm{b}$ illustrate respectively the average MSE performance for problem (8) and average sum MSE performance for problem (9) of Algorithm $1\left(k_{3}=3, I=\right.$ $\left.2, N_{t}=4\right)$. The solid lines show the performance of Algorithm 1 while the dotted lines show performances of the traditional optimization algorithm without considering the transceiver impairment. Simulation results show that the change of $k_{1}$ and $k_{2}$ has little effect on the average MSE performance for problem (8) and the average sum MSE performance for problem (9) of Algorithm 1. However, the average MSE for problem (8) and the average sum MSE performance for problem (9) without considering transceiver impairment both decrease as the value of $k_{1}$ and $k_{2}$ increases.

Figure 5 demonstrates the average sum MSE performance of Algorithm 1 for problem (9) with $N_{t}=16$ and $N_{t}=32$, respectively under varying transceiver impairments and different numbers of users in each cell $\left(k_{2}=\right.$ $8, k_{3}=3$ ). Simulation results show that Algorithm 1 for problem (9) always outperforms the traditional algorithm without considering the transceiver impairment. The performances of these algorithms all deteriorate with an increasing number of served users. In contrary, the performances of these algorithms are improved by increasing the number of transmit antennas for a fixed number of served users.

\subsection{Numerical evaluation for Algorithm 2}

The performances of the proposed multicell multiuser beamforming schemes for maximizing the system sumrate are investigated via numerical simulations. For 
(a) Convergence Trajectories of Algorithm 1 for Problem (8)

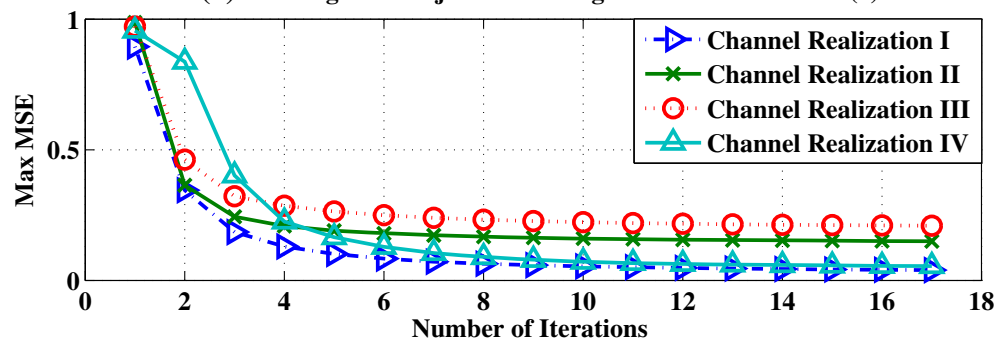

(b) Convergence Trajectories of Algorithm 1 for Problem (9)

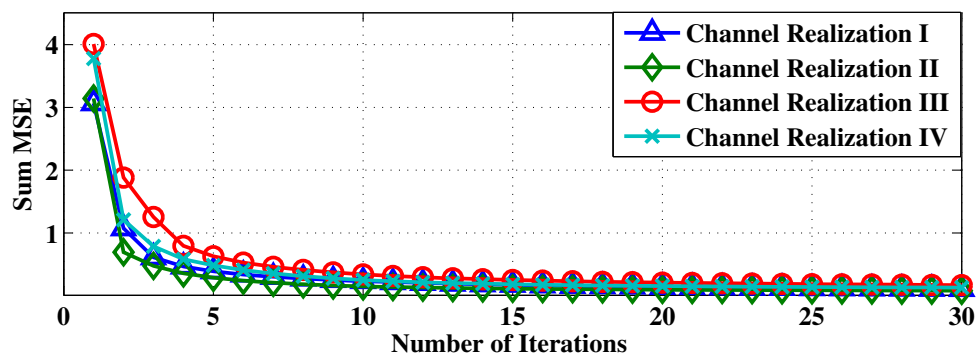

Fig. 3 Convergence trajectories of Algorithm 1. a Convergence trajectories of Algorithm 1 for problem (8). b Convergence trajectories of Algorithm 1 for problem (9)

comparison, we also evaluate the performances of the traditional optimization algorithm without considering the transceiver impairment $\left(k_{1}=0, k_{2}=+\infty, k_{3}=0\right)$.

Figure 6 shows the convergence behavior of Algorithm 2 for problem (26) under a few random channel realizations. Similar to the results in Fig. 3, it is shown that Algorithm 2 generates a monotonic non-decreasing sequence when the iteration is running, thus the convergence of Algorithm 2 can be guaranteed by the monotonic boundary theorem. Moreover, one can see that Algorithm 2 can always converge to a stable point within a couple of iterations.

Figure 7 illustrates the average system sumrate versus the coefficient $k_{1}$ of Algorithm $2\left(k_{3}=3, \delta=\right.$
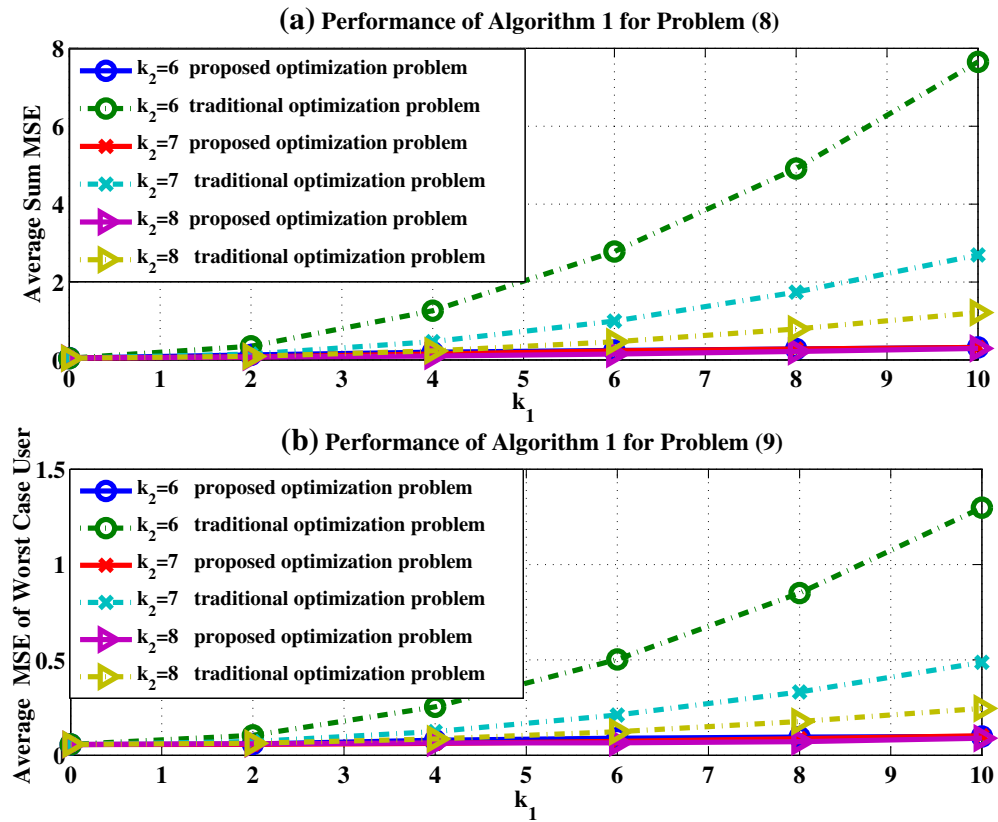

Fig. 4 Average MSE performance of Algorithm 1. a Performance of Algorithm 1 for problem (8). b Performance of Algorithm 1 for problem (9) 
(a) Performance of Algorithm 1 for Problem (9) (M=16)

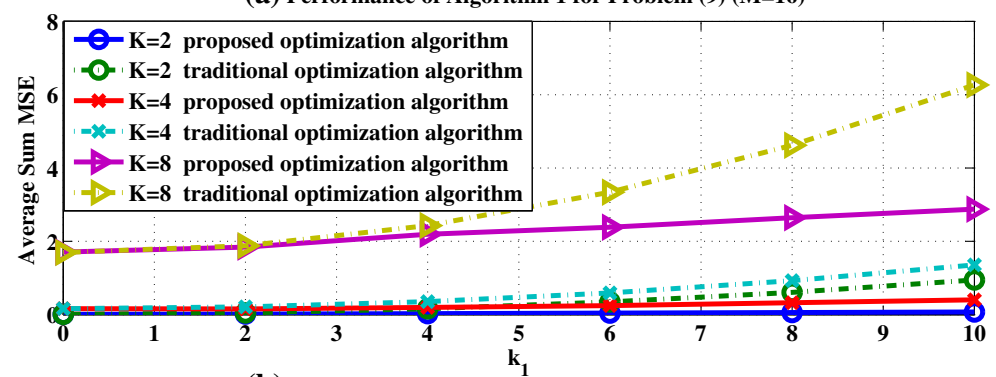

(b) Performance of Algorithm 1 for Problem (9) (M=32)

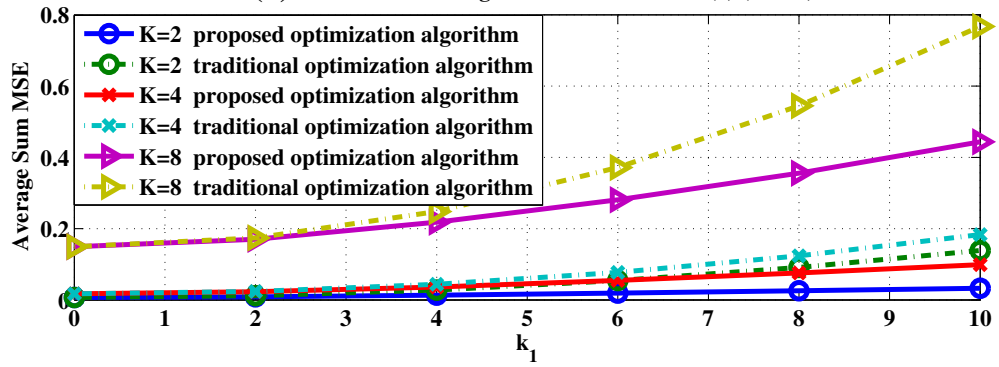

Fig. 5 Average sum MSE performance of Algorithm 1. a Performance of Algorithm 1 for problem (9) $(M=16)$. b Performance of Algorithm 1 for problem (9) $(M=32)$

0.005, $I=2, N_{t}=4$ ). Simulation results show that Algorithm 2 achieves a significant gain as compared with the traditional optimization algorithm. For the traditional scheme, the transceiver impairment becomes serious with the increasing of $k_{1}$ and decreasing of $k_{2}$, which leads to a serious deterioration of the system sumrate performance. While, for Algorithm 2, the change of $k_{1}$ and $k_{2}$ shows little effect on the system throughput which maintains a high sumrate.

Figures 8 and 9 show the system sumrate performance of Algorithm 2 varying with the coefficient $k_{1}$ and the user number $I$ under configurations $N_{t}=\{32,64\}, k_{2}=$ $8, k_{3}=3$. Simulation results show that for the traditional scheme, the increasing transceiver impairment, i.e., the

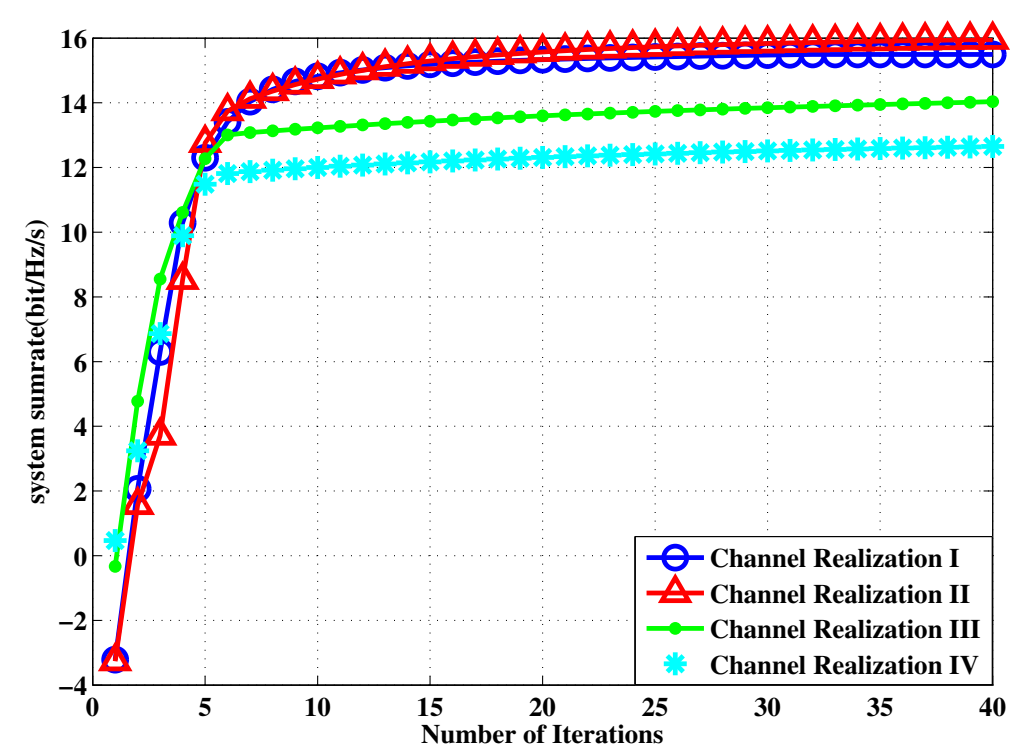

Fig. 6 Convergence trajectories of Algorithm 2 


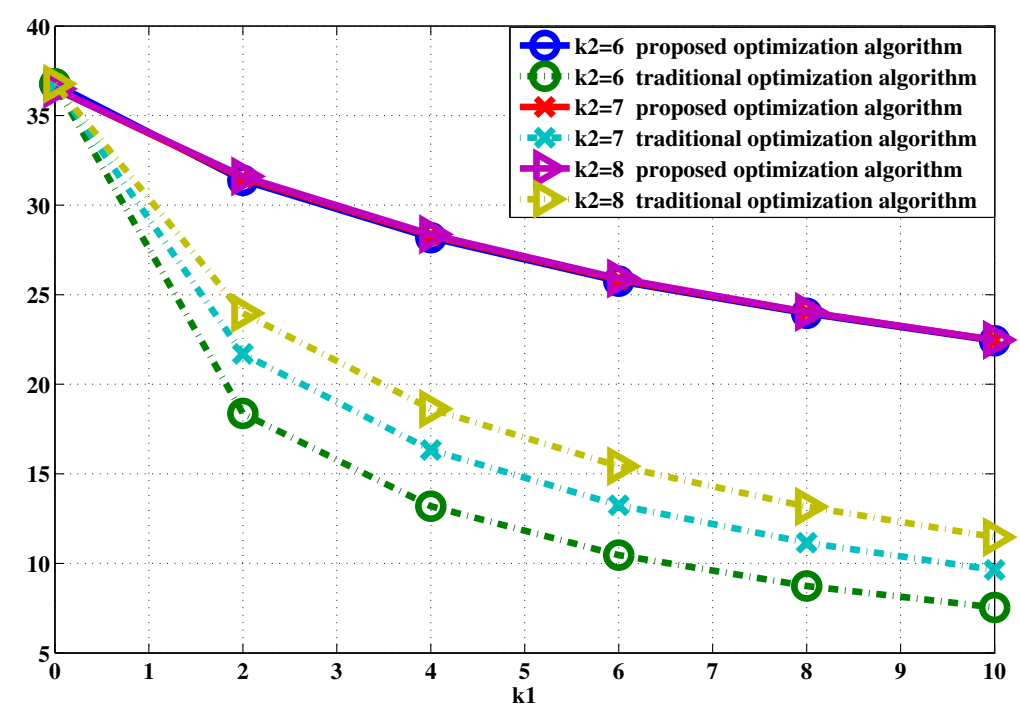

Fig. 7 Performance of Algorithm 2 for problem (26)

increase of $k_{1}$, results in an obvious performance degradation. However, the proposed sumrate maximization optimization algorithm can combat to some degree the transceiver impairment and thus shows a better sumrate performance.

\subsection{Numerical evaluation for Algorithm 3}

Finally, the performance of the proposed energy-efficient coordinated beamforming scheme is also investigated via numerical simulations. The circuit power per antenna is $P_{c}=30 \mathrm{dBm}$, and the basic power consumed at the BS is
$P_{0}=40 \mathrm{dBm}$ [38]. The transmit power budget is set to $P$ for each BS. The convergence threshold $\eta$ is set to be $10^{-3}$.

In the legends of the figures, EEMax (Aware/Ignorant) and SRMax (Aware/Ignorant) denote, respectively, the energy efficiency maximization and sum rate maximization with/without taking hardware-impaired transceivers into account.

Figure 10 illustrates the average energy efficiency of EEMax (Aware/Ignorant) and SRMax (Aware/Ignorant) with varying value of maximum transmit powers under 1000 random channel realizations. Numerical results

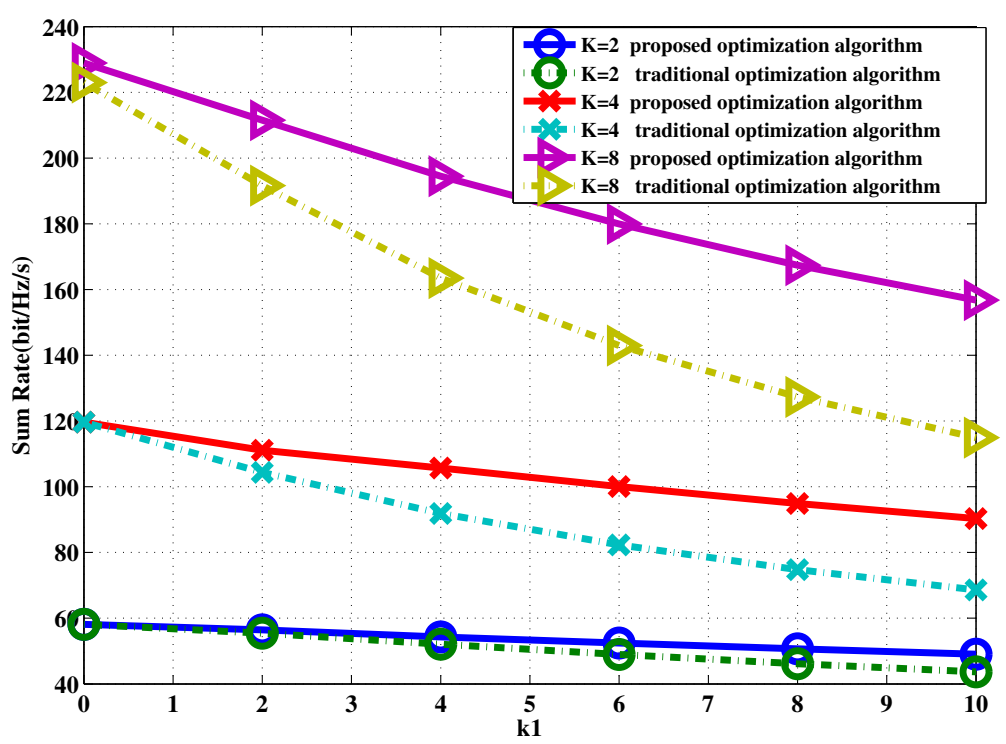

Fig. 8 Performance of Algorithm 2 for problem $(26)\left(N_{t}=32\right)$ 


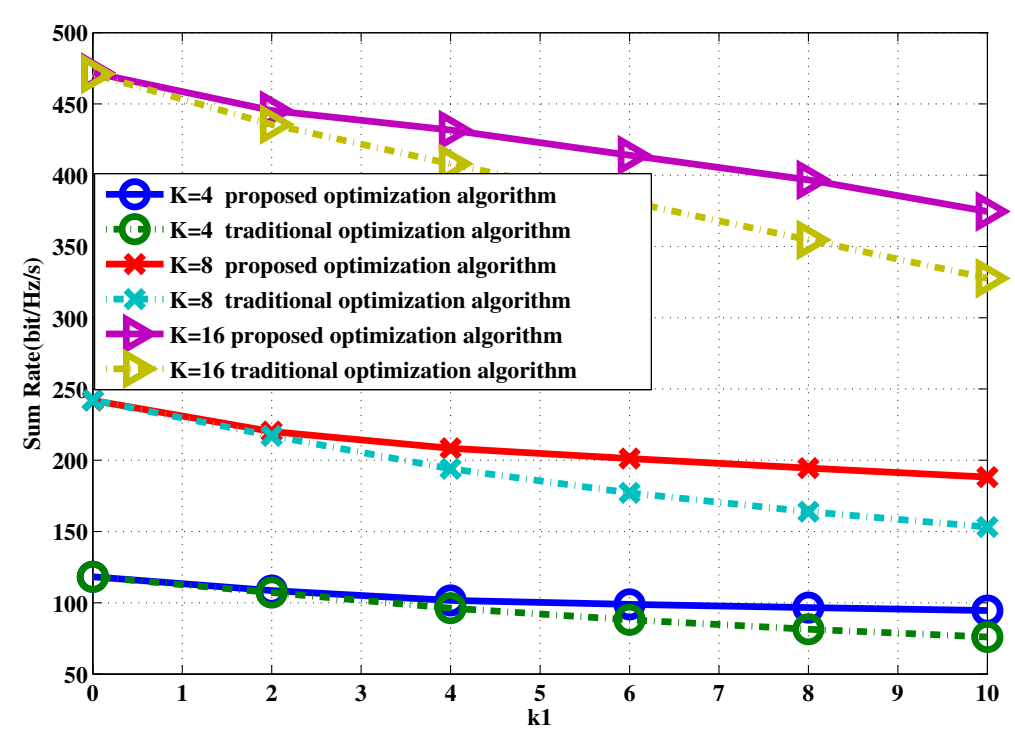

Fig. 9 Performance of Algorithm 2 for problem $(26)\left(N_{t}=64\right)$

show that the EEMax (Aware) and SRMax (Aware) algorithms outperform, respectively, the EEMax (Ignorant) and SRMax (Ignorant) algorithms in terms of energy efficiency, especially in the middle-high transmit power constraint region. This also means that the system energy efficiency performance can be improved if the energy efficient beamforming design takes into account the transceiver hardware impairment. While in the high transmit power region, it is observed that the energy efficiency achieved by EEMax (Aware/Ignorant) is better than that of SRMax (Aware/Ignorant). This is because in
SRMax(Aware/Ignorant) the capacity gain cannot compensate for the negative impact of the maximum power consumption, resulting in a low energy efficiency.

\section{Conclusions}

In this paper, we focused on the coordinated beamforming design for the multicell multiuser downlink system with transceiver impairments. We firstly derived the closedform expression of the MSE including the transceiver impairments. Then, we proposed two alternating optimization beamforming algorithms to solve the worst

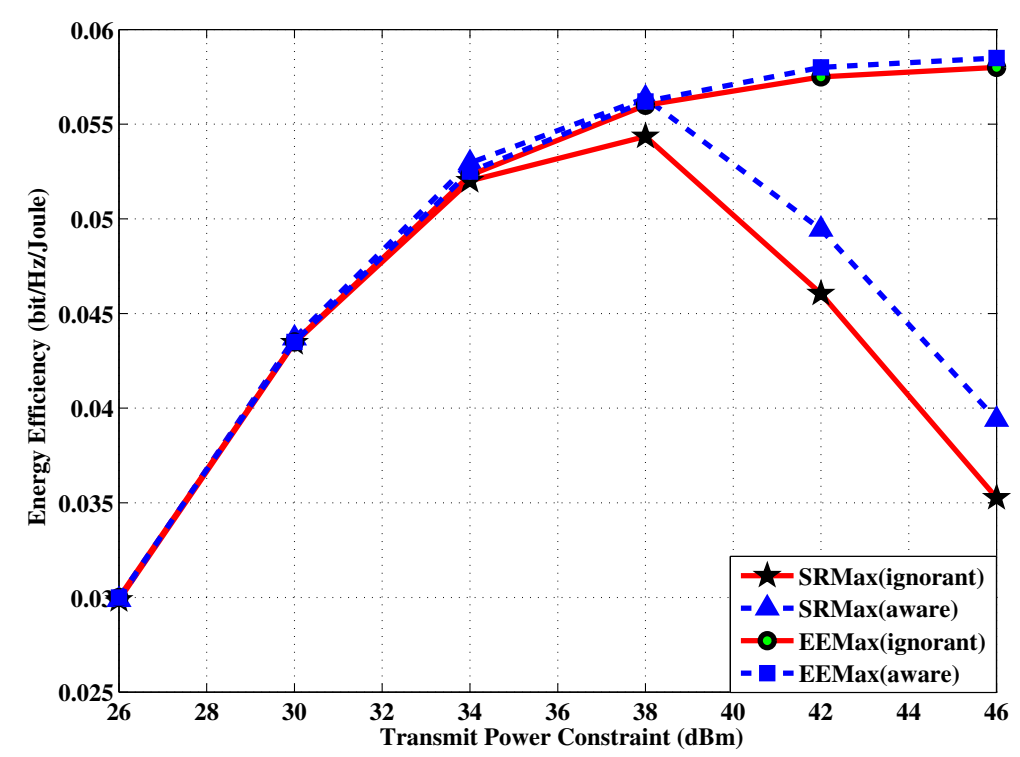

Fig. 10 Average system energy efficiency (bits/Hz/Joule) versus transmit power constraints, $M=3, N_{t}=4, I=2, \kappa_{0}=\kappa_{2}=0.1, \kappa_{1}=5.755$ 
user MSE minimization optimization problem, the sum MSE minimization problem, and the system sumrate maximization problem. Furthermore, we also devised an energy-efficient coordinated beamforming algorithm for the multicell system by taking into account transceiver impairments. The convergence of the proposed iterative algorithms was all proved. Numerical results showed that our proposed schemes obtained a better performance than the conventional algorithms which did not consider transceiver impairments in the beamforming design.

\section{Endnote}

${ }^{1}$ Here we assume that the impairments among different antennas are uncorrelated for simplicity, which has been validated in $[28,31,32]$.

\section{Competing interests}

The authors declare that they have no competing interests.

\section{Acknowledgements}

This work was supported by the National Basic Research Program of China under Grant 2013CB329002, National Natural Science Foundation of China under Grants 61471120, 61271018, 61422105, and 61372101, and Research Project of Jiangsu Province under Grants BK20130019, and BK2012021.

\section{Author details}

${ }^{1}$ School of Information Science and Engineering, Southeast University, Nanjing 210096, China. ${ }^{2}$ The State Key Laboratory of Millimeter Waves, Southeast University, Nanjing 210096, China.

Received: 24 December 2014 Accepted: 6 July 2015

Published online: 06 August 2015

\section{References}

1. 3GPP TR 36.819 v11.0.0, Coordinated multi-point operation for LTE physical layer aspects. 3GPP Technical Report (2011)

2. D Gesbert, S Hanly, H Huang, S Shamai, O Simeone, W Yu, Multi-cell MIMO cooperative networks: A new look at interference. IEEE J. Sel. Areas Commun. 28(9), 1380-1408 (2010)

3. H Dahrouj, W Yu, Coordinated beamforming for the multicell multi-antenna wireless system. IEEE Trans. Wireless Commun. 9(5), 1748-1759 (2010)

4. Y Huang, G Zheng, M Bengtsson, K Wong, L Yang, B Ottersten, Distributed multicell beamforming with limited intercell coordination. IEEE Trans. Signal Process. 59(1), 728-738 (2011)

5. E Bjornson, N Jalden, M Bengtsson, B Ottersten, Optimality properties, distributed strategies, and measurement-based evaluation of coordinated multicell OFDMA transmission. IEEE Trans. Signal Process. 59(12), 6086-6101 (2011)

6. B Clerckx, H Lee, Y Hong, G Kim, A practical cooperative multicell MIMO-OFDMA network Based on rank coordination. IEEE Trans. Wireless Commun. 12(4), 1481-1491 (2013)

7. E Bjornson, R Zakhour, D Gesbert, B Ottersten, Cooperative multicell precoding: rate region characterization and distributed strategies with instantaneous and statistical CSI. IEEE Trans. Signal Process. 58(8), 4298-4310 (2010)

8. Y Y Huang, G Zheng, M Bengtsson, K Wong, L Yang, B Ottersten, Distributed multicell beamforming design approaching pareto boundary with max-min fairness. IEEE Trans. Wireless Commun. 11(8), 2921-2933 (2012)

9. Y Huang, C Tan, B Rao, Joint beamforming and power control in coordinated multicell: max-min duality, effective network and large system transition. IEEE Trans. Wireless Commun. 12(6), 2730-2742 (2013)

10. K Manosha, S Joshi, N Rajatheva, M Latva-aho, in Proc. IEEE Global Communications Conference (GLOBECOM). Energy efficient power control and beamforming in multi-antenna enabled femtocells, (2012), pp. 3472-3477
11. Xu J, Qiu L, Energy efficiency optimization for MIMO broadcast channels IEEE Trans. Wireless Commun. 12(2), 690-701 (2013)

12. C Jiang, LJr. Cimini, Energy-efficient transmission for MIMO interference channels. IEEE Trans. Wireless Commun. 12(6), 2988-2999 (2013)

13. Y Huang, S He, S Jin, H Wang, L Yang, Duality based energy-efficient beamforming design for multiuser downlink systems. IEEE Wireless Commun. Lett. 3(4), 409-412 (2014)

14. S He, Y Huang, S Jin, F Yu, L Yang, Max-Min energy efficient beamforming for multicell multiuser joint transmission systems. IEEE Commun. Lett. 17(10), 1956-1959 (2013)

15. S He, Y Huang, S Jin, L Yang, Coordinated beamforming for energy efficient transmission in multicell multiuser systems. IEEE Trans. Commun. 61(12), 4961-4971 (2013)

16. Y Huang, S He, S Jin, W Chen, Decentralized Energy Efficient Coordinated Beamforming for Multicell Systems. IEEE Trans. Veh. Tech. 63(9), 4302-4314 (2014)

17. K Wang, T Chang, W Ma, A So, in Proc. IEEE International Conference on Acoustics, Speech and Signal Processing (ICASSP). Probabilistic SINR constrained robust transmit beamforming: A Bernstein-type in equality based conservative approach, (2011), pp. 3080-3083

18. D Ponukumati, F Gao, M Bode. Proc. IEEE Global Communications Conference (GLOBECOM), (2011), pp. 1-5

19. A Tajer, N Prasad, X Wang, Robust linear precoder design for multi-cell downlink transmission. IEEE Trans. Signal Process. 59(1), 235-251 (2011)

20. E Bjornson, G Zheng, M Bengtsson, B Ottersten, Robust monotonic optimization framework for multicell MISO systems. IEEE Trans. Signal Process. 60(5), 2508-2523 (2012)

21. T Bogale, L Vandendorpe, B Chalise, Robust transceiver optimization for downlink coordinated base station systems: distributed algorithm. IEEE Trans. Signal Process. 60(1), 337-350 (2012)

22. M Speth, SA Fechtel, G Fock, Optimum receiver design for wireless broad-band systems using OFDM-Part I. IEEE Trans. Commun. 47(11), 1668-1677 (1999)

23. T Schenk, X Tao, P Smulders, in Proc. IEEE Vehicular Technology Conference (VTC Fall). Influence and suppression of phase noise in multi-antenna OFDM, (2004), pp. 1443-1447

24. C Studer, M Wenk, A Burg, in Proc International ITG Workshop on Smart Antennas (WSA). MIMO transmission with residual transmit-RF impairments, (2010), pp. 189-196

25. N Moghadam, P Zetterberg, Händel, H Hjalmarsson, in Proc. IEEE Int. Symp. Personal, Indoor Mobile Radio Commun. (PIMRC) Correlation of distortion noise between the branches of MIMO transmit antennas, (2012), pp. 2079-2084

26. T Schenk. Proc. International Symposium on Communications, Control and Signal Processing (ISCCSP), (2008), pp. 117-122

27. G Santella, F Mazzenga, A hybrid analytical-simulation procedure for performance evaluation in M-QAM-OFDM schemes in presence of nonlinear distortions. IEEE Trans. Veh. Tech. 47(1), 142-151 (1998)

28. E Bjornson, P Zetterberg, M Bengtsson, B Ottersten, Capacity limits and multiplexing gains of $\mathrm{MIMO}$ channels with transceiver impairments. IEEE Commun. Lett. 17(1), 91-94 (2012)

29. E Bjornson, P Zetterberg, M Bengtsson, in Proc. IEEE Global Communications Conference (GLOBECOM). Optimal coordinated beamforming in the multicell downlink with transceiver impairments, (2012), pp. 4775-4780

30. C Galiotto, Y Huang, N Marchetti, M Zorzi, in Proc. European Wireless. Performance evaluation of non-ideal RF transmitter in LTE/LTE-advanced systems, (2009), pp. 266-270

31. C Studer, M Wenk, A Burg, in Proc. European Conference on Antennas and Propagation (EUCAP). System-level implications of residual transmit-RF impairments in MIMO systems, (2011), pp. 2686-2689

32. C Studer, M Wenk, A Burg, in Proc. International ITG Workshop on Smart Antennas (WSA). MIMO transmission with residual transmit-RF impairments, (2010), pp. 189-196

33. E Bjornson, E Jorswieck, Optimal resource allocation in coordinated multi-cell systems. Foundations Trends Commun. Inf. Theory. 9(2-3) 113-381 (2013)

34. H Holma, A Toskala, LTE for UMTS: evolution to LTE-advanced, Second Edition. (John Wiley \& Sons, Ltd, 2011)

35. LTE Performance vs. output power. Technical Note. [Online]. Available: http://www.minicircuits.com/app/AN60-050.pd 
36. J Bibby, Axiomatisations of the average and a further generalisation of monotonic sequences. Glasgow Math. J. 15, 63-65 (1974)

37. S Christensen, R Agarwal, E Carvalho, J Cioffi, Weighted sum rate maximization using weighted MMSE for MIMO-BC beamforming design IEEE Trans. Wireless Commun. 7(12), 4792-4799 (2008)

38. H Ngo, E Larsson, T Marzetta, Energy and spectral efficiency of very large multiuser MIMO systems. IEEE Trans. Commun. 61(4), 1436-1449 (2013)

39. S He, Y Huang, L Yang, B Ottersten, Coordinated multicell multiuser precoding for maximizing weighted sum energy efficiency. IEEE Trans. Signal Process. 62(3), 741-751 (2014)

40. R Jagannathan, On some properties of programming problems in parametric form pertaining to fractional programming. Manag. Sci. 12(7), 609-615 (1966)

41. L Grippo, M Sciandrone, On the convergence of the block nonlinear Gauss-Seidel method under convex constraints. Oper. Res. Lett. 26(3), $1-10(2000)$

42. 3GPP TR 25.996 v8.0.0 Release 8, Spatial channel model for multiple input multiple output (MIMO) simulations. 3GPP Technical Report (2009)

43. 3GPP TS 36.814 v9.0.0 Release 9, Further advancements for E-UTRA physical layer aspects. 3GPP Technical Report (2010)

\section{Submit your manuscript to a SpringerOpen ${ }^{\mathcal{O}}$ journal and benefit from:}

- Convenient online submission

- Rigorous peer review

- Immediate publication on acceptance

- Open access: articles freely available online

- High visibility within the field

- Retaining the copyright to your article

Submit your next manuscript at $\gg$ springeropen.com 